\title{
The evolution of the meaning of blood hyperviscosity in cardiovascular physiopathology: Should we reinterpret Poiseuille?
}

\author{
Sandro Forconi ${ }^{\mathrm{a}, *}$ and Tommaso Gori ${ }^{\mathrm{b}}$ \\ ${ }^{\text {a }}$ Centro Siena-Toronto, University of Siena, Siena, Italy \\ ${ }^{\mathrm{b}}$ Kardiologie, Klinikum der Johannes Gutenberg-Universität Mainz, II Medizinische Klinik, Mainz, \\ Germany \\ E-mails:forconi@unisi.it,tommaso.gori@utoronto.ca
}

In the 1960s and 1970s, a number of researchers (including ourselves) involved in the study of cardiovascular pathophysiology and particularly in the development of techniques to quantify blood flow, came across the observation that, along with vessel diameter, also blood viscosity plays an important role not only in theory but also in practice. Until then, viscosity was thought to play only a marginal role in determining blood flow, a concept which was based on the 1828 theories of Jean Louis Marie Poiseuille (Fig. 1, and [1]). ${ }^{1}$ In his well-known formula, named after its fathers Hagen ${ }^{2}$ and Poiseuille,

\footnotetext{
* Corresponding author: Sandro Forconi, MD, Professor Emeritus of Internal Medicine, President of the Centro SienaToronto, University of Siena, Siena, Italy. E-mail: forconi@ unisi.it.

${ }^{1}$ Jean Louis Marie Poiseuille was a French physician and physiologist. Poiseuille was born (April 22, 1797) in Paris, France, where he died at the age of 72 (December 26, 1869). From 1815 to 1816 he studied at the École Polytechnique in Paris. He was trained in physics and mathematics. In 1828 he earned the degree of doctor of science with a dissertation entitled "Recherches sur la force du coeur aortique" (studies on the force of the aortic heart). He was interested in understanding the principles regulating the flow of human blood in narrow tubes. In $\mathbf{1 8 3 8}$ he experimentally derived, and in $\mathbf{1 8 4 0}$ and 1846 formulated and published, the Poiseuille's law (or the Hagen-Poiseuille law also named after G.H.L. Hagen, see below) about the volume of the laminar stationary flow of incompressible uniform viscous liquid (so-called Newtonian fluid) through a cylindrical tube with a constant circular cross-section. The law was successfully applied for blood flow in capillaries and veins, for air flow in lung alveoli, for the flow through a hypodermic needle, and, more trivially but for some more interestingly, through a soda straw.
}

${ }^{2}$ Gotthilf Heinrich Ludwig Hagen (March 3, 1797-February 3, 1884) was a German physicist and hydraulic engineer. Hagen was born in Königsberg, Brandenburg, Prussia (today Kaliningrad, Russia). Independently of Poiseuille, Hagen in 1839 carried out the first carefully documented friction experiments in low-speed tube laminar flow, from which was derived the Hagen-Poiseuille law. The law is a development of the Darcy-Weisbach equation, developed in the field of hydraulics and which is otherwise valid for all types of flow. The theoretical derivation of slightly different Poiseuille's original form of the law was made independently by Wiedman in 1856 and Neumann and E. Hagenbach in $1858(1859,1860)$. Hagenbach was the first who called this law the Poiseuille's law. The law is also very important specially in hemorheology and hemodynamics, both fields of physiology. The Poiseuille's law was later in 1891 extended to turbulent flow by L.R. Wilberforce, based on Hagen- 


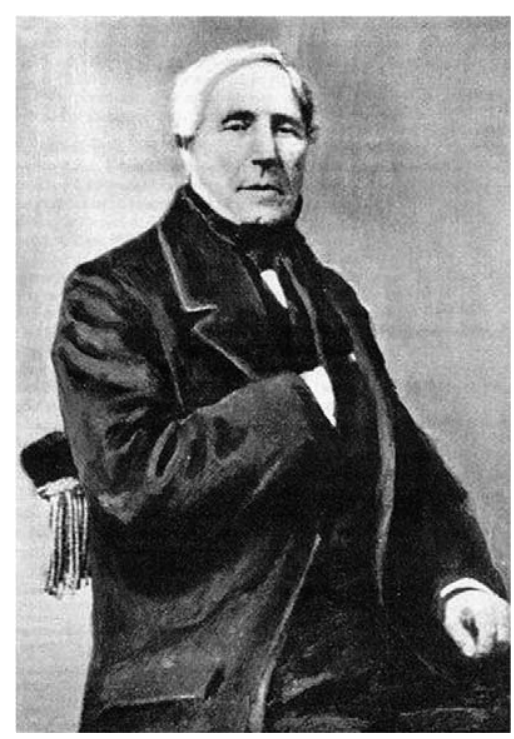

Fig. 1. Jean Louis Marie Poiseuille.

the blood flow volume through a rigid tube is directly proportional to the fourth power of the radius and inversely proportional to the viscosity of the fluid. Since very small variations in the diameter of the radius determine major variations of flow, the importance of viscosity was neglected until the early 1960s. As it happens for many things in medicine, the importance of viscosity was only understood when reliable measurement techniques were developed in the early 1960s. The first viscometers were introduced in medicine from the industry of tar, tooth paste and paints. With the introduction of these instruments, it became possible to discover the importance of variations in this parameter in both physiology and pathology [2-5]. It was probably in an excess of enthusiasm for this "new" parameter that it was postulated that conditions in which viscosity is primarily altered may be associated with cardiovascular disease (so-called "primary hyperviscosity syndromes") [4]. Subsequently, Di Perri and Forconi introduced the concept of "secondary hyperviscosity syndromes" (Fig. 2) [2,3,5]: in these conditions, while not being the cause of the disease, hyperviscosity was seen in a bidirectional way, as a marker or epiphenomenon of a reduction in blood flow caused by variations in blood diameter. Whether a cause (primary hyperviscosity syndromes) or a consequence (secondary syndromes) of disease, hyperviscosity was always associated with a negative connotation, i.e., it was seen as a parameter that, by increasing peripheral resistances, would lead to impaired blood-tissue exchanges and ischemia.

Our group has challenged this construct for many years. There are several examples that contrast the concept that hyperviscosity should be seen as a pathologic factor: for instance, hemodilution has never clearly demonstrated to improve patients' symptoms and prognosis in ischemic diseases, and hemoconcentration (for instance, that illegally induced in athletes with erythropoietin doping) definitively improves athletic performances. Further, a number of champions in long-distance running, mostly Africans, are carriers of sickle cell trait (if not disease) and, therefore, of a sclerocythemic hyperviscosity syndrome associated with reduced red blood cells deformability. The impact of these modifications in

bach's work. Curiosity. The law itself shows how an interesting field this is, because the Darcy-Weisbach equation should be properly named in full as the Chezy-Weisbach-Darcy-Poiseuille-Hagen-Reynolds-Fanning-Prandtl-Blasius-von KarmanNikuradse-Colebrook-White-Rouse-Moody equation or CWDPHRFPBKNCWRM equation in 'short' (Source: Wikipedia). 


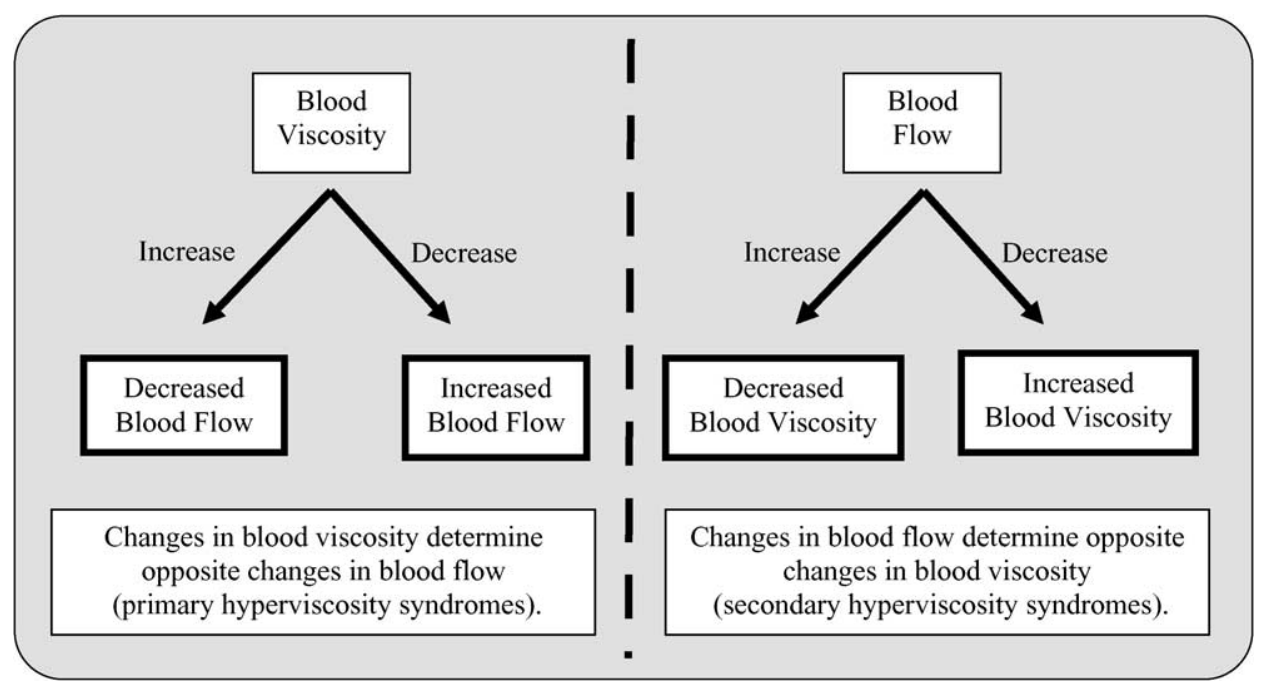

Fig. 2. The complex relationship between blood flow and viscosity in the so-called "hyperviscosity syndromes".

reducing hyperemic blood flow, seen the athletic performances of these individuals, must be definitely minor.

An important help in the understanding of these controversies came from observation on the role of the vascular endothelium in the control of vasomotion, particularly within the microcirculation $[6,7]$. While Poiseuille's law is valid only for rigid tubes, the vascular system is a continuous equilibrium between vasodilation and vasoconstriction as it effects autocrine control of vascular tone via the release of endothelial autacoids and mechanoceptor responses. Further, endothelial autacoids have not only an effect on the regulation of wall motion, but also on the deformability and aggregability of circulating cells, erythrocytes, leucocytes and platelets, thus controlling both arterial tone and blood viscosity. The complexity of this system is exemplified by the following concepts: (1) each of these autacoids has its own endogenous antagonist, often another endothelial autacoid (for instance, nitric oxide and endothelin-1 control the same functions in opposite directions); (2) the action of these endothelial and non-endothelial mediators differs dramatically with regards to its location: for instance, $\alpha$-adrenergic receptors in smooth muscle cells determine vasoconstriction, while $\beta_{3}$-adrenergic receptors in endothelial cells determine vasodilation; (3) the secretion of these mediators is not constant, and it varies within each cardiac cycle according to instantaneous variations in biochemical and mechanical forces: for instance, the secretion of nitric oxide peaks with shear stress during systole, and that of endothelin-1 behaves reciprocally. In the presence of continuous flow, this rhythm is lost [8]; (4) finally, not only circulating blood cells react to endothelial autacoids (for instance, nitric oxide reduces platelet, red blood cell and white blood cells aggregability), but they also participate in the secretion (or at least in the transport to distant vascular beds) of these otherwise chemically instable mediators.

Thus, cardiovascular homeostasis is the balance between biochemical and physical factors such as flow volume, blood pressure, wall stretch, and viscosity, as expressed by the ratio of shear stress and shear rate. Seen the complexity of these phenomena, we believe that perturbations in the release of these hemodynamically active substances should not be termed "endothelial dysfunction", as is now practice. Endothelial "function" is a complex combination of the coordinated effect of multiple substances affecting the regulation of both vascular tone and blood viscosity. This coordinated effect is often, in a simplistic approach, quantified with one single parameter, for instance diameter of a conduit artery or 
blood flow. As such, one is lead to believe that an abnormality in one parameter can be extrapolated to mean dysfunction of a complex system likely involving thousands of parameters and mediators. Defining this abnormality as overall endothelial (dys)function appears without doubt a leap of faith. For instance, we recently demonstrated that the most commonly used parameter to assess endothelial function, flow-mediated dilation, can be easily complemented by the use of the reciprocal flow-mediated constriction [9]. In these studies, we demonstrated that an impaired endothelium-mediated vasodilation might not mean "endothelial dysfunction", but, more simply, "endothelial vasomotor activation at rest" (see [9] for more details). Further, it has been shown that, while not affecting reactive hyperemia in response to ischemia, hemodilution significantly reduces the endothelium-dependent vasodilation triggered by this hyperemia [10]. While clearly not damaging the endothelium, a reduction in viscosity caused reduced endothelial vasomotor reactivity. Thus, a blunted endothelium-dependent vasodilation could be the expression of at least three phenomena: (1) impaired endothelial biochemical activity ("true" endothelial dysfunction); (2) reduced stimulus (reduced shear stress due to modifications in blood flow or viscosity); (3) endothelial activation at baseline (such that any stimulus does not produce further response).

These considerations partially explain the failure of clinical hemorheologists throughout the years, as we struggled with vascular physiology and mostly failed in our premature (often driven by pharmaceutical industry) efforts to understand the mechanisms of pathology. A number of studies were performed in an effort to demonstrate a pharmacological effect on hyperviscosity, as if it were a disease to treat. "Rheological therapies" were proposed in the assumption that "hyperviscosity equals ischemia". The above considerations lead us now to believe that terms such as "hemorheological deterioration" and "endothelial dysfunction" have been superficially used in the past, forgetting that what appears to be negative might simply be the result of a physiological, protective, response.

The introduction of some (really not many!) new, more modern and sophisticated (but not necessarily more useful), machines brought, in the last International Conferences of clinical hemorheology, a number of studies reproducing earlier results, with scarce tendency to a diverse interpretation and to a "rehabilitation" of hyperviscosity. Viscosity remains an enemy to fight against. However, along with these studies, evidence has been presented that both platelets and red blood cells are important determinants of nitric oxide release [11-15]. This confirms how complicated vascular biology is and emphasizes that we should avoid simplistic interpretations. The dynamic relationship between vascular wall and circulating cells appears to be even more complex when one considers that these cells participate in the release of factors affecting flow volume and velocity, contractility and relaxation of the vascular wall. Blood viscosity, like blood pressure, is a factor in the regulation of this balance.

Recent research has attempted to examine the response to hemoconcentration. After transfusing blood in patients with sickle cell disease, Coates observed vasodilation [16]. Accordingly, Intaglietta et al. showed that increases of hematocrit with transfusions of concentrated oxidized erythrocytes (rich in metaemoglobin and, therefore, unable to carry oxygen) lead to evidence of increased nitric production [17]. Thus, hemoconcentration - and the ensuing hyperviscosity, independently of the blood's oxygen transport capacity, may lead to increased (endothelium-dependent) blood flow and hypotension. In turn, increased oxygen extraction and blood flow would reduce tissue ischemia. Although the mechanisms of this process are yet to be understood, hyperviscosity might be one of the organism's responses to ischemia. Interestingly, by inducing release of antiaggregant and vasodilatory substances, hyperviscosity would be a self-limiting process, aimed at improving tissue perfusion only as long as ischemia persists. Thus, we believe that there is now enough evidence to see the role of hyperviscosity in cardiovascular pathology from a different perspective (Table 1, Fig. 3): hyperviscosity is not necessarily a pathology or the consequence of a pathology. Instead, it might simply be a compensatory mechanism: 
Table 1

Consequences of blood hyperviscosity

The traditional view: Hyperviscosity $>$ increased resistances $>$ decreased blood flow $>$ hypoxia (Poiseuille, 1838)

Alternate view: Hyperviscosity $>$ increased time of transit within the capillary bed $>$ increased oxygen extraction

The modern view: Hyperviscosity $>$ increased shear stress $>$ release of endothelial autacoids with activity in vasodilation/vasoconstriction, anti and pro coagulant, anti and pro fibrinolytic activity, etc.

The interaction between biochemical and physical factors may determine either increase or decrease of pressure, reduction or increase of blood flow (Forconi, 2008) in the ultimate goal of maintaining vascular homeostasis and the optimal balance between vascular offer and tissue demand of oxygen and nutrients

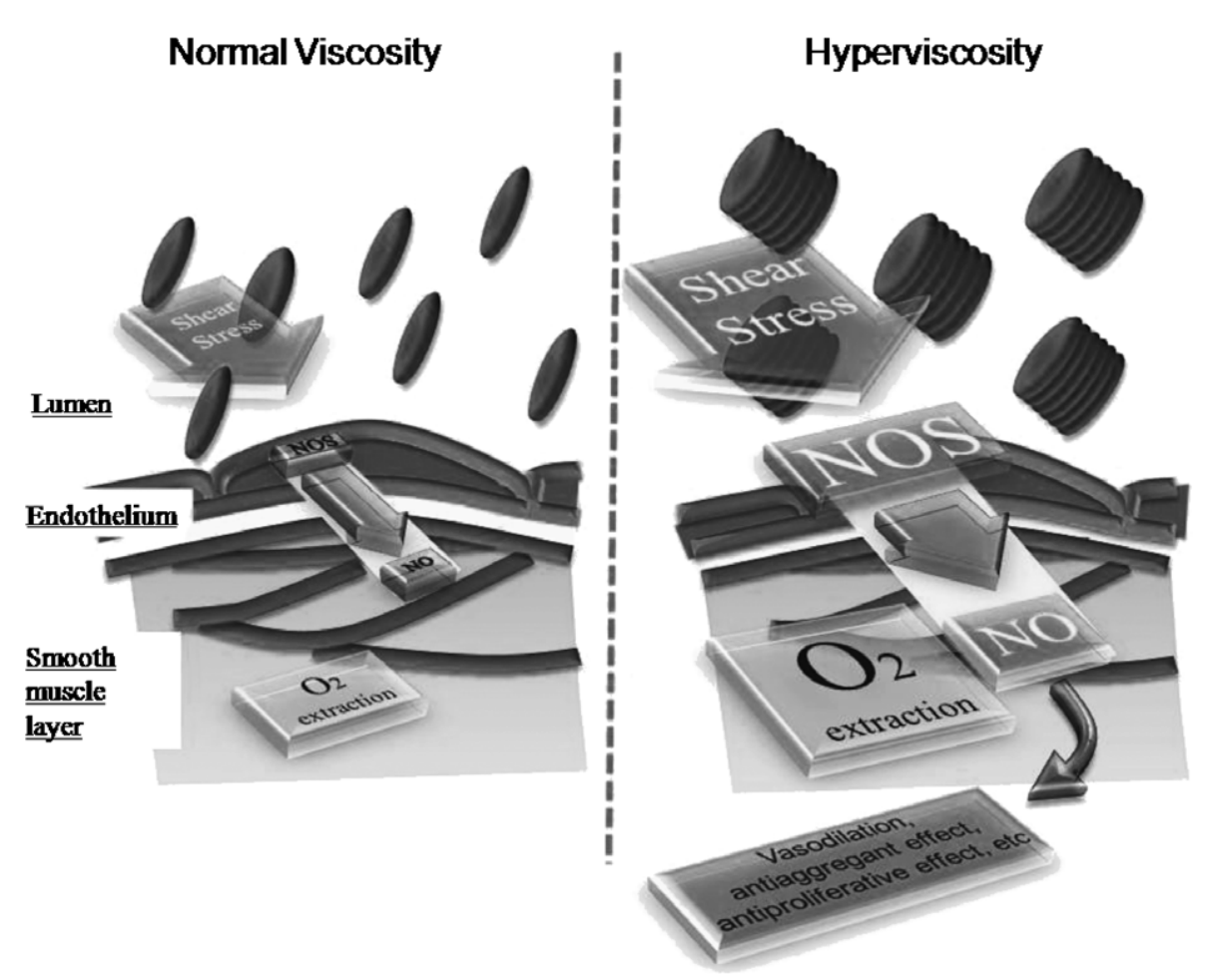

Fig. 3. Schematic representation of the hypothesis that hyperviscosity should be considered a compensatory mechanism. Besides nitric oxide (NO), shear stress is involved in the regulation of a number of other factors not represented in the figure.

by increasing the time of transit of blood through capillaries and venules, hyperviscosity would increase oxygen extraction, and favor oxygenation of ischemic tissues. Further, increased viscosity results in increased wall shear stress and recruitment of endothelial protective factors such as (but not limited to) nitric oxide.

One hundred and eighty years later, Poiseuille's law is not under discussion. However, it needs a new perspective to reconsider the positive role of hyperviscosity as a potential compensatory mechanism in the regulation of peripheral resistances and tissue perfusion in the setting of disease. This could be an interesting working hypothesis for the next years. 


\section{Acknowledgment}

The authors are grateful to Luca Trabalzini for his precious assistance.

\section{References}

[1] J.L.M. Poiseuille, Recherches sur la force du coeur aortique - thèse de médecine de Paris $\mathrm{n}^{\circ} 166,1828$.

[2] T. Di Perri, S. Forconi, F. Di Lollo and G. Pozza, Le sindromi di iperviscosità ematica: fisiologia, patologia e clinica. Il Policlinico Sez. Prat. 90 (1983), 576-584.

[3] S. Forconi, From hyperviscosity to endothelial dysfunction: a return trip?, Clin. Hemorheol. Microcirc. 30 (2004), 155165.

[4] R. Wells, Syndromes of hyperviscosity, N. Engl. J. Med. 283 (1970), 183-186.

[5] S. Forconi, D. Pieragalli, M. Guerrini, C. Galigani and R. Cappelli, Primary and secondary blood hyperviscosity syndromes, and syndromes associated with blood hyperviscosity, Drugs 33(Suppl. 2) (1987), 19-26.

[6] D. Behrendt and P. Ganz, Endothelial function. From vascular biology to clinical applications, Am. J. Cardiol. 90 (2002), 40L-48L.

[7] H.F. Galley and N.R. Webster, Physiology of the endothelium, Br. J. Anaesth. 93 (2004), 105-113.

[8] G.W. De Keulenaer, D.C. Chappell, N. Ishizaka, R.M. Nerem, R.W. Alexander and K.K. Griendling, Oscillatory and steady laminar shear stress differentially affect human endothelial redox state: role of a superoxide-producing NADH oxidase, Circ. Res. 82 (1998), 1094-1101.

[9] T. Gori, S. Dragoni, M. Lisi, G. Di Stolfo, S. Sonnati, M. Fineschi and J.D. Parker, Conduit artery constriction mediated by low flow a novel noninvasive method for the assessment of vascular function, J. Am. Coll. Cardiol. 51 (2008), 1953-1958.

[10] C. Giannattasio, A. Piperno, M. Failla, A. Vergani and G. Mancia, Effects of hematocrit changes on flow-mediated and metabolic vasodilation in humans, Hypertension 40 (2002), 74-77.

[11] J.S. Carroll, C.J. Ku, W. Karunarathne and D.M. Spence, Red blood cell stimulation of platelet nitric oxide production indicated by quantitative monitoring of the communication between cells in the bloodstream, Anal. Chem. 79 (2007), 5133-5138.

[12] B. Ozuyaman, M. Grau, M. Kelm, M.W. Merx and P. Kleinbongard, RBC NOS: regulatory mechanisms and therapeutic aspects, Trends Mol. Med. 14 (2008), 314-322.

[13] P. Kleinbongard, R. Schulz, T. Rassaf, T. Lauer, A. Dejam, T. Jax, I. Kumara, P. Gharini, S. Kabanova, B. Ozuyaman, H.G. Schnurch, A. Godecke, A.A. Weber, M. Robenek, H. Robenek, W. Bloch, P. Rosen and M. Kelm, Red blood cells express a functional endothelial nitric oxide synthase, Blood Dev. 107 (2006), 2943-2951.

[14] O. Yalcin, P. Ulker, U. Yavuzer, H.J. Meiselman and O.K. Baskurt, Nitric oxide generation by endothelial cells exposed to shear stress in glass tubes perfused with red blood cell suspensions: role of aggregation, Am. J. Physiol. Heart Circ. 294 (2008), H2098-H2105.

[15] M.T. Santos, J. Valles, A. Lago, J. Tembl, E. Sanchez, A. Moscardo and J. Cosin, Residual platelet thromboxane A2 and prothrombotic effects of erythrocytes are important determinants of aspirin resistance in patients with vascular disease, J. Thromb. Haemost. 6 (2008), 615-621.

[16] T.D. Coates, Haemorheological aspects of sickle cell disease. Symposium 26 Proc. of 13th International Congress of Biorheology and 6th International Conference on Clinical Hemorheology, Penn State University, USA, Biorheology 45 (2008), 85-87.

[17] M. Intaglietta, The physiology and benefits of increasing blood and plasma viscosity and the counterintuitive regulations of blood pressure by blood viscosity: Clinical implication. Symposium 23. Proc. of 13th International Congress of Biorheology and 6th International Conference on Clinical Hemorheology, Penn State University, USA, Biorheology 45 (2008), 77-79. 